Pacific Journal of Mathematic 


\title{
ZERO DIVISORS IN DIFFERENTIAL RINGS
}

\author{
HOWARD E. GORMAN
}

Let $R$ be a commutative ordinary differential ring with 1 . Let $A$ be a commutative differential $R$-algebra satisfying the ascending chain condition on radical differential ideals. Let $M$ be a differentially finitely generated $R$-module. We obtain the following results on the zero divisors of $A$ and $M$ in $R$. (i) If $R$ satisfies the ascending chain condition on radical differential ideals and if $A$ has zero nilradical, then the assassinator of $A$ in $R$ is finite and consists of differential ideals; it is contained in the support of $A$ in $R$, and the minimal members of each set comprise exactly the minimal prime ideals which contain the annihilator of $\mathrm{A}$ in $R$; (ii) If $R \cong A$ and $I$ is a radical differential ideal of $A$, then we obtain the assassinator of $A / I$ in $R$ from the assassinator of $A / I$ in $A$ by intersecting with $R$; (iii) If $R$ is noetherian, then the set of zero divisors of $M$ in $R$ is a unique union of prime differential ideals of $R$, each of which is maximal among annihilators in $R$ of nonzero elements of $M$; (iv) If $I$ is the annihilator or power annihilator of $M$ in $R$, then any prime ideal of $R$ minimal over $I$ is the annihilator of a nonzero element of $M$. In the above, (iii) and (iv) require an additional hypothesis to be made explicit later.

These results (except (ii)) are well known for finite modules over noetherian rings.

2. Preliminaries. In what follows, all rings are commutative and all modules are unitary. $R$ will always be a differential ring with 1, with fixed derivation denoted by " " ". By a differential module $M$ over $R$, one means an $R$-module $M$ together with an additive map from $M$ to $M$, again denoted by “' ', which satisfies $(r m)^{\prime}=$ $r^{\prime} m+r m^{\prime}$ for each $r \in R$ and $m \in M$. If $x \in M$, the successive derivatives of $x$ will be denoted by $x^{\prime}, x^{\prime \prime}, \cdots, x^{(n)}, \cdots$. By a differential algebra $A$ over $R$, one means a differential module $A$ which is a ring and for which the module derivation is a ring derivation. By an ideal of $A$, we always mean an algebra ideal.

Let $M$ be any $R$-module and $T \subseteq M$ a subset. We denote the zero divisors of $T$ in $R$ by $\mathscr{F}_{R}(T)$ and the annihilator of $T$ in $R$ by $\mathscr{A}_{R}(T)$. The assassinator of $M$ in $R$, written $\operatorname{Ass}_{R} M$, is the set of prime ideals of $R$ which are the annihilators of nonzero elements of $M$. The support of $M$ in $R$, written $\operatorname{Supp}_{R} M$, is the set of prime ideals $P$ of $R$ such that $M_{P} \neq 0$.

Now let $R$ be a differential ring and $M$ a differential $R$-module. 
Denote by $[T] / R$ the smallest differential submodule of $M$ containing $T$. We call $M d$-finitely generated if there exists $n \geqq 0$ and $x_{1}, \cdots$, $x_{n}$ in $M$ such that $M=\left[x_{1}, \cdots, x_{n}\right] / R$.

Let $S \subseteq R$ be a multiplicatively closed set with $0 \notin S$. Then the derivations on $R$ and $M$ extend by the usual quotient formula to make $M_{s}$ into a differential $R_{s}$-module. (See [2; Lemma 1].)

Assume, in addition, that $M$ is a differential $R$-algebra. Denote by $\{T\} / M$ the smallest radical differential ideal containing $T$. The following fact is a trivial consequence of [5; Lemma 1.3]. Let Rad $M=0$ (i.e., $M$ has zero nilradical), and let $T$ be a subset of either $R$ or $M$. Then $\mathscr{A}_{R}(T)$ and $\mathscr{A}_{M}(T)$ are radical differential ideals.

3. The assassinator. We begin by stating the first main theorem.

THeorem 1. Let $R$ be a differential ring and $A$ a differential $R$-algebra. Let $R$ and $A$ satisfy the ascending chain condition on radical differential ideals, and let $\operatorname{Rad} A=0$. Then $\operatorname{Ass}_{R} A$ is finite, consists of differential prime ideals, and is contained in $\operatorname{Supp}_{R} A$. The minimal members of each of these sets are the same and coincide with the prime ideals of $R$ minimal over $\mathscr{A}_{R}(A)$.

Before proving Theorem 1, we need a series of lemmas.

Lemma 1. Let $R$ be a differential ring satisfying the ascending chain condition on radical differential ideals. Let $A$ be a nonzero differential $R$-algebra with $\operatorname{Rad} A=0$. Then $\operatorname{Ass}_{R} A \neq \varnothing$.

Proof. For any nonzero $a \in A, \mathscr{Z}_{R}(a)$ is a proper, radical differential ideal of $R$. By hypothesis, there are ideals of $R$ maximal among annihilators of nonzero elements of $A$. That these ideals are prime is well known [4; Theorem 6].

Lemma 2. Let $R$ be a differential ring, and let $M$ be a differential $R$-module. Let $T$ be a subset of $M$, and suppose that $\mathscr{A}_{R}(T)$ is a differential ideal. Then:

(i) $\mathscr{A}_{R}(T)=\mathscr{A}_{R}([T] / R)$;

(ii) if $M$ is a differential $R$-algebra, then $\mathscr{A}_{R}(T)=\mathscr{A}_{R}([T] / M)$; if, in addition, $\operatorname{Rad} M=0$, then $\mathscr{A}_{R}(T)=\mathscr{A}_{R}(\{T\} / M)$.

Proof. Let $y \in \mathscr{A}_{R}(T)$. Then, since $x y^{\prime}+x^{\prime} y=0$ for any $x \in T$, and $y^{\prime} \in \mathscr{A}_{R}(T)$, we see that $x^{\prime} y=0$. Hence, $x^{\prime \prime} y+x^{\prime} y^{\prime}=0$. The above argument applied to $y^{\prime}$ instead of to $y$ would have resulted in $x^{\prime} y^{\prime}=0$. Hence $x^{\prime \prime} y=0$. Continuing in this way, we see that 
$x^{(k)} y=0$ for each nonnegative integer $k$. Now since an arbitrary element of $[T] / R$ has the form $\sum_{i, j} a_{i, j} x_{j}{ }^{(i)}$, and an arbitrary element of $[x] / M$ (when $M$ is an $R$-algebra) has the form $\sum_{i, j} b_{i j} x_{i}^{(i)}+\sum_{i, j} c_{i j} x_{j}{ }^{(i)}$, for $b_{i j} \in M$ and $a_{i j}, c_{i j} \in R$, for every $i$ and $j$, we see that $\mathscr{A}_{R}(T) \cong$ $\mathscr{A}_{R}([T] / R)$ and $\mathscr{A}_{R}(T) \subseteq \mathscr{A}_{R}([T] / M)$. Since the opposite inclusions are clear, we have equality.

Now assume that $M$ is an $R$-algebra and that $\operatorname{Rad} M=0$. By the above, we will be through once we show that $\mathscr{A}_{R}([T] / M) \subseteq$ $\mathscr{A}_{R}(\{T\} / M)$. Now $\mathscr{A}_{M}\left(\mathscr{A}_{R}([T] / M)\right)$ is a radical differential ideal of $M$. Since it contains $T$, it contains $\{T\} / M$; i.e., $\{T\} / M$ annihilates $\mathscr{A}_{R}([T] / M)$; therefore, $\mathscr{A}_{R}([T] / M)$ annihilates $\{T\} / M$. This completes the proof.

Lemma 3. Let $R$ be a differential ring, and let $A$ be a differential $R$-algebra satisfying the ascending chain condition on radical differential ideals, and such that $\operatorname{Rad} A=0$. Let $P$ be a prime ideal of $R$ containing $\mathscr{A}_{R}(A)$. Then $P \in \operatorname{Supp}_{R}$ A.

Proof. Since $A$ satisfies the ascending chain condition on radical differential ideals, there must be $a_{1}, \cdots, a_{r}$ in $A$ such that $A=\left\{a_{1}\right.$, $\left.\cdots, a_{r}\right\} / A$. Suppose that $A_{P}=0$. Then there are $s_{i} \in R-P$ such that $s_{i} a_{i}=0$ for each $i$. Let $s=\prod_{i=1}^{r} s_{i}$. Then $s a_{i}=0$ for each $i$. Since $\operatorname{Rad} A=0, \mathscr{Z}_{A}(s)$ is a radical differential ideal of $A$ containing each $a_{i}$, and so must equal $A$. But then $s A=0$; i.e., $s \in \mathscr{A}_{R}(A)$, which contradicts $s \notin P$. This completes the proof.

Lemma 4. Let $R$ be a differential ring satisfying the ascending chain condition on radical differential ideals, and let $A$ be a differential $R$-algebra with Rad $A=0$. Then $\operatorname{Ass}_{R} A \subseteq \operatorname{Supp}_{R} A$, and each member of $\operatorname{Supp}_{R} A$ contains a member of $\operatorname{Ass}_{R} A$. In particular, both sets have the same minimal elements.

Proof. That $\operatorname{Ass}_{R} A \subseteq \operatorname{Supp}_{R} A$ is just [1; $\S 1,{ }^{\circ} 3$, Prop. 7(i)]. Now let $Q \in \operatorname{Supp}_{R} A$. Then $A_{Q} \neq 0$ as an $R_{Q}$-algebra. By Lemma 1, $\operatorname{Ass}_{R_{Q}}\left(A_{Q}\right) \neq \varnothing$. Let $P_{1} \in \operatorname{Ass}_{R_{Q}}\left(A_{Q}\right)$ with $P_{1}=\mathscr{L}_{R_{Q}}(a / 1)$. Since Rad $\left(A_{Q}\right)=0, P_{1}$ is a differential ideal. Let $P=\left\{r \in 1 \mid r / 1 \in P_{1}\right\}$. Then $P$ is a prime differential ideal of $R$ and $P \subseteq Q$. We claim that $P \in \operatorname{Ass}_{R} A$. By hypothesis, $P=\left\{p_{1}, \cdots, p_{n}\right\} / R$ for some $p_{1} \cdots, p_{n} \in R$. Since $p_{i} a / 1$ $=0$, there are $s_{i} \in R-P$ such that $p_{i} s_{i} a=0$ for each $i$. Hence, if $s=\prod_{i=1}^{n} s_{i}, p_{i} \in \mathscr{K}_{R}(s a)$ for each $i$. Since $\mathscr{Z}_{R}(s a)$ is a radical differential ideal of $R, P \subseteq \mathscr{Z}_{R}(s a)$. On the other hand, if $x \in \mathscr{Z}_{R}(s a)$, then $x a / 1=0$; i.e., $x / 1 \in P_{1}$; i.e., $x \in P$. Hence $P=\mathscr{Z}_{R}(s a) \in \operatorname{Ass}_{R} A$, and we are done. 
Lemma 5. Let $R$ be a differential ring, and let $A$ be a differential $R$-algebra satisfying the ascending chain condition on radical differential ideals. Assume that $\operatorname{Rad} A=0$. Then $A$ has a normal series

$$
A=A_{0} \supseteqq A_{1} \supseteqq \cdots \supseteqq A_{n}=0
$$

where

(i) $A_{i}$ is a radical differential ideal of $A$ for each $i$;

(ii) $\mathscr{A}_{R}\left(A_{i-1} / A_{i}\right)=\operatorname{Ass}_{R}\left(A_{i-1} / A_{i}\right)$ for each $i$, and both consist of a single prime differential ideal $P_{i}$ of $R$.

Proof. Let $B \neq A$ be a radical differential ideal of $A$. Then $A / B$ is a differential $R$-algebra satisfying the ascending chain condition on radical differential ideals, and $\operatorname{Rad}(A / B)=0$. Since $A / B \neq 0$, we are guaranteed by Lemma 1 that there exists in $\operatorname{Ass}_{R}(A / B)$ a differential prime ideal $P=\mathscr{\varkappa}_{R}(x)(x \in A / B$ and nonzero) which is maximal among the annihilator of nonzero elements of $A / B$. Let $B_{1}=\varphi^{-1}(\{x\} /$ $(A / B)$ ) where $\varphi$ is the canonical homomorphism of $A$ onto $A / B$. Then $B_{1}$ is a radical differential ideal of $A, B \varsubsetneqq B_{1}$ and $B_{1} / B \cong\{x\} /(A / B)$ so that $\mathscr{A}_{R}\left(B_{1} / B\right)=P$ by Lemma 2. Now suppose that $Q \in \operatorname{Ass}_{R}\left(B_{1} / B\right)$. Then $Q=\mathscr{Z}_{R}\left(b_{1}\right)$ for some $b_{1} \in B_{1} / B$. Since $P b_{1}=0, P \leqq Q$; hence, by the maximality of $P, P=Q$ and $A \mathrm{ss}_{R}\left(B_{1} / B\right)$ consists of the single prime $P$.

Starting with $B=0$ and using the above method, we construct an increasing chain of radical differential ideals of $A$ satisfying the conclusions of the lemma. By hypothesis, this chain must stop; i.e., at some stage, $B_{1}=\mathrm{A}$, and we are done.

Proof of Theorem 1. We follow the notation of Lemma 5. By $\left[1 ; \S 1,{ }^{\circ} 1\right.$, Prop. 3],

$$
\operatorname{Ass}_{R} A \cong \cup_{i=1}^{n} \operatorname{Ass}_{R}\left(A_{i-1} / A_{i}\right)=\left\{P_{1}, \cdots, P_{n}\right\}
$$

so that $\operatorname{Ass}_{R} A$ is finite and consists of differential ideals. By Lemma 4, $\operatorname{Ass}_{R} A \subseteq \operatorname{Supp}_{R} A$, and each has the same minimal elements. (In fact, since $P_{i} \in \operatorname{Supp}_{R}\left(A_{i-1} / A_{i}\right)$ by Lemma 4 and since $0 \neq\left(A_{i-1} / A_{i}\right)_{P_{i}}=$ $\left(A_{i-1}\right)_{P_{i}} /\left(A_{i}\right)_{P_{i}}$ each $P_{i} \in \operatorname{Supp}_{R} A_{i-1} \subseteq \operatorname{Supp}_{R} A$.) That these minimal elements coincide with the prime ideals of $R$ minimal over $\mathscr{A}_{R}(A)$ follows from the following two facts: The minimal elements of $\operatorname{Ass}_{R} A$, and so of $\operatorname{Supp}_{R} A$, contain $\mathscr{A}_{R}(A)$; the primes minimal over $\mathscr{A}_{R}(A)$ are members of $\operatorname{Supp}_{R} A$ by Lemma 3. This completes the proof.

CoRollary. Let the hypotheses be as in Theorem 1. Then $\operatorname{Supp}_{R} A$ consists of exactly the prime ideals of $R$ which contain $\mathscr{A}_{R}(A)$. 
We remark that if $R$ contains the rational numbers and satisfies the ascending chain condition on radical differential ideals, then any quotient by a differential ideal of the differential polynomial ring over $R$ in a finite number of differential indeterminates also satisfies the ascending chain condition on radical differential ideals.

If we assume that $R \subseteq A$, we get the following result with no chain condition assumptions on $R$.

THEOREM 2. Let $R$ be a differential ring contained in the differential $R$-algebra $A$. Assume that $A$ satisfies the ascending chain condition on radical differential ideals. Let $I$ be a radical differential ideal of $A$. Then: (i) I can be written uniquely as $I=\cap_{i=1}^{n} P_{i}$ where the $P_{i}$ are prime differential ideals of $A$; (ii) if $Q_{i}=P_{i} \cap R$, then

$$
A \mathrm{ss}_{A}(A / I)=\left\{P_{1}, \cdots, P_{n}\right\} \text { and } \operatorname{Ass}_{R}(A / I)=\left\{Q_{1}, \cdots, Q_{n}\right\} \text {. }
$$

Proof. We note that (i) is well known and proved more directly in [5; Theorem 7.5]. Now $A / I$, viewed as an $A$-algebra, satisfies the hypotheses of Lemma 1 and Theorem 1 . Let $P_{1}, \cdots, P_{n}$ be the unique elements of $\operatorname{Ass}_{A}(A / I)$ minimal over $\mathscr{A}_{A}(A / I)$. Since $1 \in A, \mathscr{A}_{A}(A / I)=$ $I$, and since $I$ is a radical ideal, $I=\bigcap_{i=1}^{n} P_{i}$. This proves (i).

Since the $P_{i}$ are minimal over $\mathscr{A}_{A}(A / I)$, they are minimal members of $\operatorname{Ass}_{A}(A / I)$ by Theorem 1. On the other hand, let $P=\mathscr{Z}_{A}\left(a_{1}\right) \in$ $\operatorname{Ass}_{A}(A / I)$, with $a_{1} \in A / I$. Let $a \in A$ be mapped to $a_{1}$. Then $a \notin P_{j}$ for some $j=1, \cdots, n$. But $P a \subseteq I \subseteq P_{j}$, so that $P \subseteq P_{j}$; i.e., $P=$ $P_{j}$. Hence $\operatorname{Ass}_{A}(A / I)=\left\{P_{1}, \cdots, P_{n}\right\}$.

Now let $P_{i}=\mathscr{Z}_{A}\left(a_{i}\right), a_{i} \in A / I$ for each $i$. Then $Q_{i}=P_{i} \cap R$ must be $\mathscr{\varkappa}_{R}\left(a_{i}\right)$ for each $i$; i.e., $Q_{i} \in \operatorname{Ass}_{R}(A / I)$.

To complete the proof, we must show that any $Q \in \operatorname{Ass}_{R}(A / I)$ is one of the $Q_{i}$. Localize $A$ and $R$ at $Q$. Then $A_{Q}$ is an $R_{Q}$-algebra satisfying the hypotheses of the theorem and $I_{Q}$ is a radical differential ideal of $A_{Q}$. Further, $I_{Q}$ is a proper ideal of $A_{Q}$ for, since $I \cap$ $R \cong Q$, we see that $(I \cap R)_{Q}=I_{Q} \cap R_{Q} \subseteq Q_{Q}$; i.e., $R_{Q} \nsubseteq I_{Q}$. Since each $P_{i}$ is prime, $I_{Q}=\left(\cap_{i=1}^{n} P_{i}\right)_{Q}=\cap_{i=1}^{r}\left(P_{i}\right)_{Q}$ where we have assumed that $P_{1}, \cdots, P_{r}$ are exactly those among $P_{1}, \cdots, P_{n}$ such that $\left(P_{i}\right)_{Q} \neq A_{Q}$. Note that $r>0$ by Lemma 1 since $A_{Q} / I_{Q} \neq 0$. By the initial argument in this part of the theorem, $\operatorname{Ass}_{R_{Q}}\left(A_{Q} / I_{Q}\right)=\left\{\left(P_{1}\right)_{Q}, \cdots,\left(P_{r}\right)_{Q}\right\}$. Since $Q_{Q} \subseteq \mathscr{Z}_{R_{Q}}\left(A_{Q} / I_{Q}\right), Q_{Q} \subseteq\left(P_{i}\right)_{Q} \cap R_{Q}=\left(Q_{i}\right)_{Q}$ for some $i$. Since $Q_{Q}$ is maximal, $Q_{Q}=\left(Q_{i}\right)_{Q}$; i.e., $Q=Q_{i}$, and the proof is complete.

4. The case for modules. The situation for modules is less complete. However, under the restriction given below, we can gain some information about $\operatorname{Ass}_{R}(M)$ when $M$ is a $d$-finitely generated $R$-module. 
We say that the differential $R$-module $M$ satisfies the property (\#) if ideals of $R$ maximal among the annihilators of nonzero elements of $M$ are differential ideals. We say that $M$ satisfies the property (\#\#) if $M / N$ satisfies the property (\#) for every differential submodule $N$ of $M$.

THEOREM 3. Let $R$ be a noetherian differential ring and $M a$ nonzero, d-finitely generated $R$-module which satisfies the property (\#\#). Then $\operatorname{Ass}_{R} M$ is finite.

Proof. The assassinator of nonzero modules over noetherian rings is never empty. Using the condition (萧) and Lemma 2(i), we modify the proof of Lemma 5 to prove an analogue of Lemma 5 in which the $A_{i}$ are replaced by differential $R$-modules. The result now follows as in the first part of Theorem 1 .

Further progress in this direction is limited by the fact that prime ideals of $R$ containing $\mathscr{A}_{R}(M)$ need not be in $\operatorname{Supp}_{R} M$. The correct modification is given in Lemma 7. (For example, let $R=Z$, the integers, with the trivial derivation. Let $M$ be generated over $Z / 2 Z$ by 1 and the set $\left\{x / 2^{n}\right\}$ for $n=0,1,2, \cdots$, and have derivation defined by $\left(x / 2^{n}\right)^{\prime}=x / 2^{n+1}$. Then $M=[1, x] / Z$. Now $\mathscr{A}_{Z}(M)=0$; but if $P=3 Z, M_{P}=0$.)

The following discussion indicates what is still true if we assume only the condition $(\#)$. We shall need the result $[2 ;$ Th. 1$]:{ }^{1}$

THEOREM A. Let $R$ be a noetherian differential ring, and let $M$ be a d-finitely generated $R$-module. Then $M$ satisfies the ascending chain condition on differential submodules.

\section{We can now prove}

THEOREM 4. Let $R$ be a noetherian differential ring and $M$ a $d$-finitely generated $R$-module which satisfies the property (\#). Then $\mathscr{\varkappa}_{R}(M)$ is expressible uniquely as the union of a finite number of differential prime ideals, each of which is maximal among the annihilators of nonzero elements of $M$.

Proof. Each nonzero $x \in M$ has an annihilator ideal, and $\mathscr{\varkappa}_{R}(M)$ is clearly their union. Each such annihilator is contained in a maximal one which is prime, and differential by assumption. Let $\left\{P_{\lambda}\right\}_{\lambda \in A}$ be the set of these maximal annihilators, and let $P_{\lambda}=\mathscr{Z}_{R}\left(x_{\lambda}\right), x_{\lambda} \in M$

1 This theorem, in different language, is originally due to J. Johnson, Differential dimension polynomials and a fundamental theorem on differential modules, Amer. J. Math., 91 (1969), 239. 
for each $\lambda \in \Lambda$. The differential submodule $N$ of $M$ generated by the $x_{\lambda}$ 's is $d$-finitely generated by Theorem A. Let $N=\left[x_{1}, \cdots, x_{n}\right] / R$, with the $x_{1}, \cdots, x_{n}$ chosen from among the $x_{\lambda}$ 's. Then, for any $\lambda$, $x_{\lambda}=\sum_{i, j} r_{i j} x_{i}^{(j)}$, with $r_{i j} \in R$ for each $i$ and $j$, and only a finite number of values for $j$ appearing. Since, by Lemma $2, P_{i}=\mathscr{A}_{R}\left(\left[x_{i}\right]\right)$ for each $i=1,2, \cdots, n$, this implies that $P_{\lambda} \supseteq \cap_{i=1}^{n} P_{i}$. This implies, by maximality, that $P_{\lambda}$ is one of the $P_{i}^{\prime}$ 's. Hence, $\mathscr{Z}_{R}(M)=\cup_{i=1}^{n} P_{i}$.

To show uniqueness, we remark that if $Q$ were a member of another such union, then $Q \subseteq \cup_{i=1}^{n} P_{i}$ implies that $Q$ equals one of the $P_{i}$ 's $[4 ;$ Th. 8]. This proves the theorem.

For any $R$-module $M$, define $\mathscr{P} \mathscr{X}_{R}(M)$, the power annihilator of $M$ in $R$, to be the set of $r$ in $R$ such that for every $m \in M$, there is a positive integer $n$ with $r^{n} m=0$. Then $\mathscr{P}_{R}(M)$ is an ideal which contains both $\mathscr{A}_{R}(M)$ and its radical. (If $M$ is finitely generated, it equals this radical.)

Lemma 6. Let $M$ be a differential $R$-module. Let $a \in M$ and $r \in$ $R$, and suppose that $r a=0$. Then, for every nonnegative integer $n$, we have $r^{n+1} a^{(n)}=0$.

Proof. We proceed by induction, the case $n=0$ being satisfied by hypothesis.

If $r^{n} a^{(n-1)}=0$, then $r^{n} a^{(n)}+n r^{n-1} r^{\prime} a^{(n-1)}=0$.

On multiplying through by $r$, we have the result.

Lemma 7. Let $R$ be a differential ring $M$ a d-finitely generated $R$-module. Let $P \subseteq R$ be a prime ideal containing $\mathscr{P}_{\mathscr{A}}(M)$. Then $M_{P} \neq 0$.

Proof. Let $M=\left[m_{1}, \cdots, m_{r}\right] / R$, and assume that $M_{P}=0$. Then there is an $s \in R-P$ such that $s m_{i}=0$ for each $i$. By Lemma 6, $s^{k} m_{i}^{(k-1)}=0$ for each $i$ and $k$, and so, for every $m \in M$, there is a positive integer $t$ with $s^{t} m=0$; i.e., $s \in \mathscr{P}_{\mathscr{A}}(M)$. This contradicts $s \notin P$.

Lemma 8. Let $M$ be any $R$-module. Let $I=\mathscr{A}_{R}(M)$ (resp., $I=$ $\mathscr{P} \mathscr{A}_{R}(M)$ ), and let $P$ be a prime ideal of $R$ containing $I$. Assume that $M_{P} \neq 0$. Then $I_{P} \subseteq \mathscr{A}_{R_{P}}\left(M_{P}\right) \subseteq P_{P}\left(\right.$ resp., $\left.I_{P} \subseteq \mathscr{P}_{\mathscr{A}_{R_{P}}}\left(M_{P}\right) \subseteq P_{P}\right)$.

Proof. The first inclusion is clear in both cases. We prove the second inclusion, $\mathscr{P} \mathscr{A}_{R_{P}}\left(M_{P}\right) \subseteq P_{P}$. Let $x / t \in \mathscr{P}_{\mathscr{R}_{P}}\left(M_{P}\right)$ with $x \in R$ and $t \in R-P$, and let $m \in M$ be such $m / 1 \neq 0$. If $(x / t)^{r} m / 1=0$, then there is an $s \in R-P$ with $s x^{r} m=0$. If $x \notin P$, then $s x^{r} \in R-P$, so 
that $s x^{n} m=0$ implies that $m / 1=0$, a contradiction. Hence, $x \in P$, and we are done.

If $M$ is any $R$-module, it is well known that any prime ideal of $R$ minimal over $\mathscr{A}_{R}(M)$ is contained in $\mathscr{Z}_{R}(M)$ (See [4; Th. 84]). A minor variant of the proof in the reference proves.

Lemma 9. Let $M$ be any $R$-module. Let $P$ be a prime ideal minimal over $\mathscr{P}_{\mathscr{A}}(M)$. Then $P \subseteq \mathscr{Z}_{R}(M)$.

THEOREM 5. Let $R$ be a noetherian differential ring and $M a$ $d$-finitely generated $R$-module. Let $I=\mathscr{A}_{R}(M)\left(\right.$ resp., $\left.\mathscr{P}_{R}(M)\right)$, and let $P$ be a minimal prime ideal over $I$. Assume that $M_{P} \neq 0$ (note Lemma 7 in this regard) and that $M_{P}$ satisfies the property (\#). Then $P$ is a differential ideal and $P \in \operatorname{Ass}_{R} M$.

Proof. $M_{P}$ is a nonzero, $d$-finitely generated module over $R_{P}$. Since $P$ is minimal over $I$, Lemma 8 implies that $P_{P}$ is minimal over $\mathscr{A}_{R_{P}}\left(M_{P}\right)$ (resp., $\left.\mathscr{P} \mathscr{A}_{R_{P}}\left(M_{P}\right)\right)$. By Lemma 9 and the remark preceding it, $P_{P} \subseteq \mathscr{L}_{R_{P}}\left(M_{P}\right)$. It follows from Theorem 4 and the maximality of $P_{P}$ that there is an $x \in M$ such that $P_{P}=\mathscr{\varkappa}_{R_{P}}(x / 1)$. Further, $P_{P}$ is a differential $R_{P}$-ideal. Since $P=\left\{r \in R \mid r / 1 \in P_{P}\right\}, P$ is a differential ideal also. Since $P_{P}$ is finitely generated, this implies the existence of an $s \in R-P$ such that $s P x=0$. But then $P=\mathscr{Z}_{R}(s x)$. For if $y s x=0$, for some $y \in R$, then $(y / 1)(x / 1)=0$; i.e., $y / 1 \in P_{P}$. It follows that $y \in P$, and we are done.

EXAMPLE. Let $S$ be a noetherian ring containing the rational numbers and equipped with the trivial derivation. Let $R$ be the ring of formal power series over $S$ in the indeterminate $z$, equipped with the derivation defined by $z^{\prime}=z$. Since every prime ideal of $R$ is of the form $P R$ or $P R+z R$, where $P$ is a prime ideal of $S, R$ satisfies the condition (\#隹) for any $R$-module. Let $x$ be an indeterminate, and let $M_{1}=R\left[x^{-1}\right]$, viewed as a differential $R$-module by the derivation $(x)^{\prime}=r$ for some unit $r \in S$. Since $x^{-(n+1)}=\left(x^{-1}\right)^{(n)}$ times a unit of $S, M_{1}$ is d-finitely generated over $R$ by 1 and $x^{-1}$. Let $M$ be any quotient module of $M_{1}$ by a differential submodule. Then $M$ and $R$ satisfy the hypotheses of Theorems 3,4 , and 5 . Notice that if $M_{1}$ is considered as a ring, Rad $M_{1}$ need not be zero.

\section{REFERENCES}

1. N. Bourbaki, Algebre Commutative, Chap. 4, Hermann, Paris, 1961.

2. H. E. Gorman, Differential rings and modules, to appear in Scripta Math.

3. - Radical regularity in differential rings, Canadian J. Math., XXIII No. 2 (1971), 197-201. 
4. I. Kaplansky, Commutative Rings, Allyn and Bacon, 1970.

5. — An Introduction to Differential Algebra, Hermann, Paris, 1957.

6. A. Seidenberg, Differential ideals in rings of finitely generated type, Amer. J. Math., 89 (1967), 22-42.

Received March 17, 1970 and in revised form April 29, 1971. The work on this paper was partially supported by NSF Grant 14023.

STANFORD UNIVERSITY 



\section{PACIFIC JOURNAL OF MATHEMATICS}

\section{EDITORS}

H. SAMELSON

Stanford University

Stanford, California 94305

C. R. HobBY

University of Washington

Seattle, Washington 98105
J. DugundjI

Department of Mathematics

University of Southern California

Los Angeles, California 90007

RICHARD ARENS

University of California

Los Angeles, California 90024

\section{ASSOCIATE EDITORS}

E. F. BECKENBACH

B. H. NeUMaNN

F. WOLF

K. YoshidA

\section{SUPPORTING INSTITUTIONS}

UNIVERSITY OF BRITISH COLUMBIA

CALIFORNIA INSTITUTE OF TECHNOLOGY

UNIVERSITY OF CALIFORNIA

MONTANA STATE UNIVERSITY

UNIVERSITY OF NEVADA

NEW MEXICO STATE UNIVERSITY

OREGON STATE UNIVERSITY

UNIVERSITY OF OREGON

OSAKA UNIVERSITY
UNIVERSITY OF SOUTHERN CALIFORNIA STANFORD UNIVERSITY

UNIVERSITY OF TOKYO

UNIVERSITY OF UTAH

WASHINGTON STATE UNIVERSITY UNIVERSITY OF WASHINGTON

$\stackrel{*}{*} \stackrel{*}{*} \stackrel{*}{*}$ AMERICAN MATHEMATICAL SOCIETY
NAVAL WEAPONS CENTER 


\section{Pacific Journal of Mathematics}

Vol. 39 , No. 1

May, 1971

Charles A. Akemann, A Gelfand representation theory for $C^{*}$-algebras ....

Sorrell Berman, Spectral theory for a first-order symmetric system of

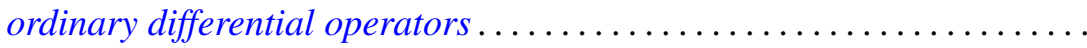

Robert L. Bernhardt, III, On splitting in hereditary torsion theories ........

J. L. Brenner, Geršgorin theorems, regularity theorems, and bounds for determinants of partitioned matrices. II. Some determinantal identities ..........................................

Robert Morgan Brooks, On representing $F^{*}$-algebras .............. 51

Lawrence Gerald Brown, Extensions of topological groups........... 71

Arnold Barry Calica, Reversible homeomorphisms of the real line ........ 79

J. T. Chambers and Shinnosuke Oharu, Semi-groups of local Lipschitzians in

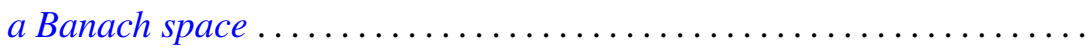

Thomas J. Cheatham, Finite dimensional torsion free rings .............

Byron C. Drachman and David Paul Kraines, A duality between

transpotence elements and Massey products ...................

Richard D. Duncan, Integral representation of excessive functions of a

Markov process ......................................

George A. Elliott, An extension of some results of Takesaki in the reduction

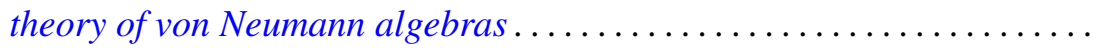

Peter C. Fishburn and Joel Spencer, Directed graphs as unions of partial

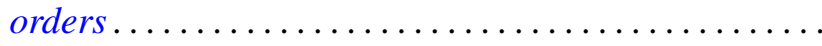

Howard Edwin Gorman, Zero divisors in differential rings ...

Maurice Heins, A note on the Löwner differential equations...

Louis Melvin Herman, Semi-orthogonality in Rickart rings. .

David Jacobson and Kenneth S. Williams, On the solution of linear G.C.D.

equations

Michael Joseph Kallaher, On rank 3 projective planes ... . .

Donald Paul Minassian, On solvable $O^{*}$-groups ...........

Nils Øvrelid, Generators of the maximal ideals of $A(\bar{D})$

Mohan S. Putcha and Julian Weissglass, A semilattice decomposition into

semigroups having at most one idempotent ............

Robert Raphael, Rings of quotients and $\pi$-regularity ....

J. A. Siddiqi, Infinite matrices summing every almost periodic sequence. .

Raymond Earl Smithson, Uniform convergence for multifunctions ...

Thomas Paul Whaley, Mulitplicity type and congruence relations in

universal algebras...

Roger Allen Wiegand, Globalization theorems for locally finitely generated modules... 